\title{
Enfoque sistémico-cibernético en el diseño de sistema de gestión de una institución de educación superior universitaria
}

\section{Systemic-cybernetic approach in the design of a management system of a university higher education institution}

\author{
Taipe-Castro, Robensoy [(D) 0000-0001-7520-5703]1 \\ 'Universidad Nacional del Centro del Perú, Huancayo, Perú \\ $\triangle$ rtaipe@uncp.edu.pe \\ Recibido: 31/10/202I; \\ Aceptado: 30/II/202I; \\ Publicado: 20/01/2022
}

Resumen: La investigación incide en el diseño del sistema de gestión en la Institución de Educación Superior Universitaria (IESU) con fines de acreditación mediante el enfoque sistémico-cibernético. El propósito es detallar la construcción del sistema de gestión basada en procesos. La metodología de diseño sistémico-cibernético es utilizada enfocada a atender criterios provenientes de la normatividad pertinente a la IESU y estandarización de sistemas de gestión. Los resultados fueron el modelo de sistema viable de la facultad, los procesos agrupados en funciones sistémicas y el mapa de procesos. Asimismo, la investigación evidencia la viabilidad organizacional de la IESU y concluye que el enfoque sistémicocibernético aborda la organización esclareciendo sus fines y medios organizacionales deseables e idealizados, ello incide en el diseño del sistema de gestión propio y particular de la institución.

Palabras clave: acreditación; cibernética organizacional; holística; mapa de procesos

Abstract: The research affects the design of the management system in the "university higher education institution" (IESU) for accreditation purposes through the systemic-cybernetic approach. The purpose is to detail the construction of the process-based management system. The systemic-cybernetic design methodology is used focused on meeting criteria from the relevant regulations of the IESU and standardization of management systems. The results were the Faculty's viable system model, the processes grouped into systemic functions and the process map. Likewise, the research shows the organizational viability of the IESU and concludes that the systemic-cybernetic approach addresses the organization by clarifying its desirable and idealized organizational goals and means, which affects the design of the institution's own and particular management system.

Keywords: accreditation; holistic; organizational cybernetics; process map

Cómo citar / Citation: Taipe-Castro, R. (202I). Enfoque sistémico-cibernético en el diseño de sistema de gestión de una institución de educación superior universitaria. Revista científica de sistemas e informática, 2(I), e250. https://doi.org//0.51252/rcsi.v2il.250

(c) Los autores. Este artículo es publicado por la Revista científica de sistemas e informática de la Universidad Nacional de San Martín. Este es un artículo de acceso abierto, distribuido bajo los términos de la Licencia Creative Commons Atribución 4.0 Internacional (https://creativecommons.org/licenses/by/4.0/deed.es) que permite Compartir (copiar y redistribuir el material en cualquier medio o formato), Adaptar (remezclar, transformar y construir a partir del material) para cualquier propósito, incluso comercialmente. 


\section{Introducción}

El diagnóstico para la formulación de la Política Nacional de la Educación Superior y TécnicoProductivo del Perú, evidencia la situación actual del problema público y la situación futura deseada de la educación superior y técnico-productiva (MINEDU, 2020).

En el árbol de problemas de la educación superior y técnico-productiva (ESTP), la población con inadecuadas competencias para ejercer su profesión y desarrollar investigación e innovación genera baja competitividad y desarrollo sostenible del país, a raíz de las siguientes causas directas: bajo acceso de la población a la ESTP, débil proceso formativo integral de la ESTP, débil articulación para el aseguramiento de la calidad de la ESTP, y por último, insuficiente movilización de recursos para asegurar la calidad y el desarrollo de la investigación e innovación de la ESTP (MINEDU, 2020).

La interrelación e interdependencia de las causas directas relacionadas con la ESTP condiciona la operación de la institución de educación superior en particular, inciden en su acceso, componentes, dirección y la interrelación con su entorno. La cuestión es ¿Cómo de forma integral, la institución de educación superior universitaria (IESU) debe enfrentar la problemática de la ESTP?

Asimismo, cabe señalar que los procesos administrativos de la universidad en el Perú evidencian una baja calificación en la satisfacción por parte de los estudiantes y egresados; además, los resultados negativos fueron: "los trámites generales" (I5.2\%), "la atención del personal administrativo" (19.8\%), y finalmente, "los procesos de matrícula e inscripciones de asignaturas" (28.3\%) (MINEDU, 2020). En base a ello, cabe preguntar ¿Qué sistema de gestión propio de la institución de educación superior universitaria incrementaría el nivel de satisfacción de los estudiantes universitarios y egresados?

El sistema de gestión de una institución es el conjunto de componentes interrelacionados - que interactúan para establecer políticas, objetivos y procesos para lograr fines organizacionales. Cada institución u organización tiene una manera de realizar sus actividades, es decir, su propio sistema de gestión para el logro de sus fines, sin embargo, en algunas oportunidades son poco eficientes, en tal caso, impera un motivo de diseño y mejora continua desde un enfoque sistémico.

Durante las últimas décadas, el diseño organizacional desde el enfoque sistémicocibernético, ha experimentado un crecimiento considerable. Además, ha interrelacionado con los diseños de sistemas de gestión.

La metodología y diseño de sistemas ha surgido como un concepto y principios de razonamiento para integrar la resolución de problemas de ingeniería tradicional con la teoría de sistemas, la ciencia de la gestión, la teoría de decisiones del comportamiento y los enfoques de planificación y diseño (Nadler, 1985). La ingeniería de sistemas combina aspectos sistémicos y sistemáticos, evidencia la práctica del pensamiento sistémico en el diseño de sistemas, su núcleo es la consideración holística del ciclo de vida completo del sistema con un fuerte enfoque en las primeras fases de desarrollo, como la identificación de las necesidades de las partes interesadas (Bretz, Könemann, Anacker, \& Dumitrescu, 2020).

Durante aproximadamente 40 años, se ha afirmado que la cibernética y el diseño tienen mucho en común. Esto se expresó originalmente a través de criterios de comunicación y 
mediante el uso de enfoques cibernéticos clásicos como métodos de uso en el diseño. Hay una relación mucho más estrecha entre la cibernética y el diseño, mediante la consideración de los desarrollos en cibernética que no estaban disponibles hace 40 años (cibernética de segundo orden) y mediante el examen de la actividad en el corazón del acto de diseño, mientras que muchos intentos anteriores se han centrado en la investigación que es mucho más sobre evaluación, prescripción y proscripción (Glanville, 2007).

Los conocimientos generales de la (gestión) cibernética (por ejemplo, viabilidad, atenuación y amplificación) pueden realizarse en las organizaciones mediante su diseño estructural. Tener una estructura que permita a las organizaciones atenuar y amplificar es una condición crucial para la viabilidad organizacional (Vriens \& Achterbergh, 20I I).

El marco de ideas basado en la cibernética organizacional y en especial el modelo de sistema viable, no solo presenta una explicación detallada de los principios de un sistema de educación superior viable, sino que también proporciona la base para un rediseño completo que puede utilizarse para ayudar a la dirección de las principales universidades a diagnosticar y mantener la viabilidad de sus sistemas. El proceso de gestión de un sistema de educación superior es complejo debido al problema de la burocracia y sus costos relacionados (Saeed Rezk \& Gamal, 2019).

El pensamiento sistémico, según lo escrito y practicado por Russell Ackoff, C. West Churchman, Peter Checkland y otros (Ackoff, 2006), (Churchman, 1995), (Checkland, 2019), contenía muchos de los impulsos que motivan la aplicación de ideas de diseño a la estrategia, la organización, la sociedad y la gestión. Ideas como involucrar a un amplio conjunto de partes interesadas, ir más allá de las métricas y cálculos simples, considerar opciones idealizadas y usar escenarios para explorarlas, cambiar los límites para replantear los problemas, la iteración, el uso liberal de diagramas e imágenes enriquecidas, y la búsqueda incansable de una mejor un conjunto de alternativas estaban todas allí (Collopy, 2019).

Por otro lado, la educación superior es fundamental para las sociedades por su responsabilidad de crear y difundir conocimiento y contribuir al desarrollo comunitario (UNESCO, 2017). La educación superior es la segunda etapa del sistema educativo nacional y consolida la formación integral de las personas, "produce conocimiento, desarrolla investigación e innovación y forma profesionales en el más alto nivel de especialización y perfeccionamiento en todos los campos del saber, el arte, la cultura, la ciencia y la tecnología, a fin de atender la demanda de la sociedad y contribuir al desarrollo sostenible del país”, Art. 49 de la Ley 28044 , (MINEDU, 2003).

Una estrategia para el diseño de sistema de gestión es abordada por el enfoque sistémicocibernético. El enfoque sistémico es una forma de pensar acerca del sistema total y sus componentes. De hecho, puede ser bastante radical para alguien pensar primero en el objetivo general y luego comenzar a describir el sistema en términos de ese objetivo general. Además, hay algunos principios para el "enfoque sistémico": “I) El enfoque de sistemas comienza cuando primero ves el mundo a través de los ojos de otro; 2) El enfoque de sistemas continúa descubriendo que cada visión del mundo está terriblemente restringida; 3) No hay expertos en el enfoque de sistemas; 4) El enfoque de sistemas no es una mala idea" (Churchman, The systems approach, 1968). El enfoque sistémico permite el acceso a lo complejo y lo admite sin desbaratar su integridad como tal. Entonces, "ve la cosa en el juego de sus interrelaciones" (Flores \& 
Ludueña, 1983). El “enfoque sistémico", es un marco de trabajo basado en la teoría de sistemas y la cibernética. Este esfuerzo, proporciona un aparato formal para tratar con sistemas complejos de todo tipo y, por lo tanto, es adoptado cada vez más en muchos campos de investigación. La teoría de sistemas y la cibernética también son reconocidas como un nuevo "lenguaje", que permite la interacción sinérgica entre diferentes disciplinas, aumentando así la posibilidad de intervenciones innovadoras y transdisciplinarias a problemas complejos (Schwaninger, 2009). En su forma más avanzada, el "enfoque sistémico" fomenta el empleo de una variedad de metodologías en combinación para gestionar "desordenes" y "problemas perversos" (Jackson, 2019) en las organizaciones y fenómenos complejos.

La Cibernética Organizacional aplica los principios relacionados con la "Comunicación y el Control" a las organizaciones, fue desarrollado por Stafford Beer (Beer, 1985). Los aspectos resaltantes de su teoría son: el concepto de viabilidad, variedad, Ley de Ashby y sus alcances, Teorema de "Conant-Ashby", atenuación y amplificación. En la Cibernética Organizacional hasta ahora ha habido un apoyo metodológico limitado para hacer un uso efectivo de este conjunto de conocimientos. El trabajo de Stafford Beer, en particular su libro "Diagnóstico del sistema para organizaciones" publicado en 1985, ofrece una guía para aplicar el modelo de sistema viable, pero no una epistemología para comprender las organizaciones (Espejo \& Reyes, 20ll). La Cibernética Organizacional es muy superior a las alternativas que normalmente ofrece la teoría de la organización (clásica, relaciones humanas y contingentes), además de ser capaz de dar sentido a los últimos hallazgos en teoría organizacional e integrarlos en una herramienta de gestión aplicable. Su enorme poder explicativo en comparación con el análisis habitual llevado a cabo en la teoría de la organización reside en la ciencia de la cibernética y la epistemología estructuralista en la que se basa. La teoría organizacional (al menos la teoría basada en el positivismo) generalmente basa sus argumentos en las relaciones observables entre fenómenos superficiales, mientras que la cibernética puede explicar estas relaciones sobre la base de procesos funcionales en el nivel profundo y estructural (Pérez Ríos, 2012). Del mismo modo, Pérez Ríos (2012), explica la gran aplicación del "Modelo de Sistema Viable" (MSV) en la Cibernética Organizacional. EI MSV describe "las condiciones necesarias y suficientes para que una organización sea viable. Cuando éstas no se cumplen, las secuelas, según la gravedad de la privación, es un desatinado funcionamiento e incluso el desvanecimiento de la organización.

El MSV intenta brindar las condiciones necesarias y suficientes de viabilidad en la que el sistema bajo estudio reconozca su propia identidad $y$, al hacerlo, invita ese reconocimiento por parte de otros. Cualquier sistema es un fenómeno subjetivo, reconocido y, en ese proceso, modificado por sus observadores, que se incluye a sí mismo. Está incrustado en un entorno con el que interactúa todo el tiempo, en los intercambios que interpretamos como modos de aprendizaje, de adaptación, de evolución (Beer, 1990). Puesto en un lenguaje más figurativo, el MSV ayuda a descifrar el "ADN" de las organizaciones, es decir, las "funciones de vida" y los principios de gobierno que son necesarios para que sean viables. Quien haya entendido el MSV descubrirá cuántos fenómenos y procesos en las organizaciones pueden explicarse, y con qué rapidez y profundidad son analizados los problemas de una organización y predecir las consecuencias de las reorganizaciones y reestructuraciones.

Así, el trabajo pretende abordar diferentes estrategias basados en el enfoque sistémicocibernético para diseñar el sistema de gestión en la institución de educación superior 
universitaria, con varios niveles de intensidad, profundidad y autenticidad entre los distintos subsistemas.

\section{Materiales y métodos}

El desarrollo de la metodología de investigación es mediante el diseño sistémico - cibernético (Beer, 1985), (Taipe Castro R., 2018).

La investigación, al inicio, reflexiona un estudio sistémico-cibernético de la institución, y luego, realiza el diseño del sistema organizativo.

El diseño sistémico-cibernético, inicia con la descripción del desdoblamiento de complejidad; luego, el diseño de autonomía y viabilidad, diseño del balance interno, diseño del balance con el entorno, diseño del componente de identidad y política organizacional; en seguida, el modelo del sistema viable, análisis de funciones sistémicas, diseño de procesos agrupados en funciones sistémicas y al final, el mapa de procesos (Taipe Castro R. M., 2009a), (Taipe Castro R. M., 2009b), (Taipe Castro R. , 2009c).

El tipo de investigación es Tecnológico aplicativo, el nivel de investigación es explicativo, es decir, "está dirigido a responder por las causas de los eventos y fenómenos bajo estudio; también, centra su propósito en explicar por qué ocurre un fenómeno y en qué condiciones se manifiesta” (Hernández Sampieri, Fernández Collado, \& Baptista Lucio, 20I4, pág. 98).

El diseño de investigación es un caso de estudio contemporáneo, mediante observador participante. El estudio de caso, según Vieytes (2004) citado por (Hurtado de Barrera, 20 I0, pág. 737), los estudios de caso "consisten en recopilar e interpretar detalladamente la mayor cantidad de información posible sobre una persona, una institución, una empresa." "El estudio de caso trata de adquirir una comprensión relativamente completa de la unidad de análisis. La cual es considerada holísticamente en lugar de indagar aspectos separados" (Hurtado de Barrera, 2010, pág. 738).

\section{Resultados y discusión}

El diseño del sistema de gestión mediante el enfoque sistémico cibernético evidencia el modelo del sistema viable, diseño de procesos agrupados en funciones sistémicas y al final, el mapa de procesos.

\section{I. Modelo de sistema viable de la Facultad de Ingeniería}

El modelo de sistema viable de la Facultad de Ingeniería, explica la integración e interrelación de los 5 sistemas, a saber: Sistema I (operación), Sistema 2 (coordinación), Sistema 3 (Cohesión), Sistema 3* (Auditoría - Monitoreo), Sistema 4 (adaptación - inteligencia) y Sistema 5 (identidad - política) (ver Fig. I).

El modelo de sistema viable, Figura I, muestra el diseño de los 3 procesos misionales, sistema I (representado por o), ellos son: Enseñanza - Aprendizaje, Investigación, Extensión Cultural y Proyección Social, entre cada uno de estos procesos misionales existe información, comunicación, insumos y requerimientos detallados en el gráfico representado como una liga entre cada proceso, esta "liga" es de color anaranjado. Cada proceso misional tiene su respectivo 
entorno identificado, al que tienen que brindar su atención mediante mecanismos atenuadores y amplificadores, a su vez cada proceso misional tiene su respectiva gestión local (representado por a) y su coordinación local en íntima relación como la de compartir datos, indicadores de gestión, requerimientos, cumplimiento de responsabilidades, esta información es llevada a la coordinación central a través de mecanismos diseñados tales como la Programación Académica, Jornadas de Investigación, Reuniones de difusión de requerimientos de Extensión Cultural, Proyección Social y Transferencia Tecnológica, mediante estos mecanismos se determinan consensos para dar atención a las necesidades requeridas por cada proceso, así como también dan cuenta de las metas que están alcanzando, toda esta información de forma resumida es llevada a la Coordinación Central; también en el gráfico, el sistema 2, estabilización y sincronización (representado por $\mathbf{\Delta}$ ), toma en cuenta la información brindada por cada uno de los procesos misionales y las decisiones de coordinación para el logro de la viabilidad de la Facultad mediante la Comisión de Estabilización y Sincronización. El sistema 3*, auditoría y monitoreo (representado por $\nabla$ ), este monitoreo es de forma esporádica y no es punitiva, tiene la finalidad de ver por otros medios no formales las causas de algunos indicadores ya sea favorable o desfavorable para la Facultad, el sistema 3, cohesión (representado por $\square$ ), es el encargado de garantizar los insumos logísticos para llevar a cabo un óptimo desarrollo de los procesos misionales, el sistema 4, Adaptación e Inteligencia (representado por $\square$ ) es el encargado de velar por los nuevos escenarios que van presentándose a la Facultad y proponer nuevos métodos y metodologías para afrontar estos cambios del entorno y el Sistema 5, Identidad y Política (representado por $\square$ ), es el sistema que brinda la capacidad de seguir realizando las actividades acorde a la identidad de la Facultad. 
Figura I. Modelo de sistema viable de Facultad (diseño)

Propósito: Brindar el servicio de formación profesional e investigación a través de la enseñanza aprendizaje, investigación y extensión cultural y proyección social para coadyuvar al desarrollo regional y nacional.

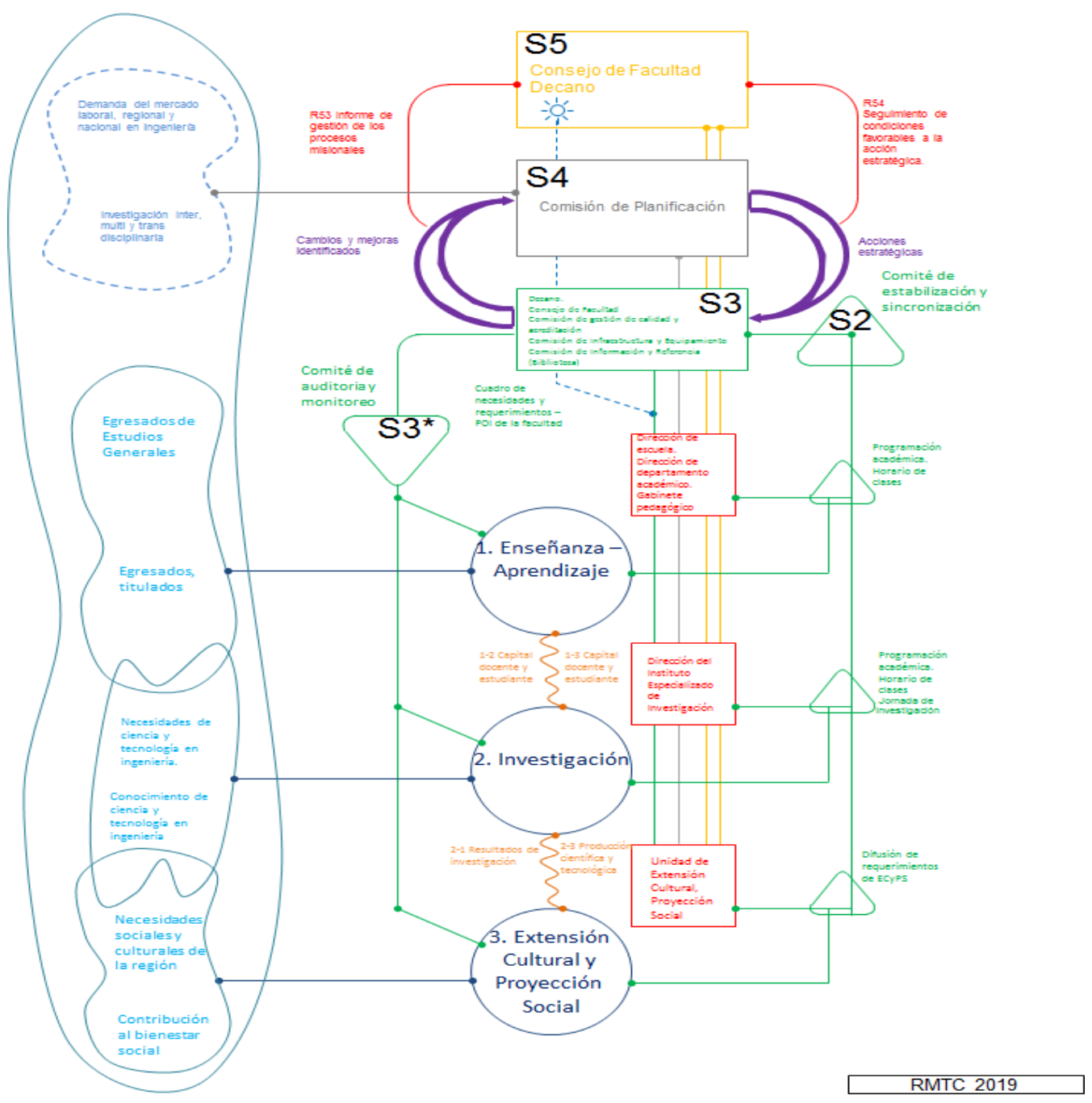

\subsection{El diseño de procesos, agrupados en funciones sistémicas}

El Diseño de Procesos, congregados en Funciones Sistémicas es de la siguiente forma:

- La función sistémica misional, sistema I, está compuesto por el Macroproceso de Formación Integral.

- La función sistémica de estabilización y sincronización, sistema 2, está compuesto por el Proceso de Estabilización y Sincronización.

- La función sistémica de cohesión, sistema 3, está compuesto por: Proceso Talento Docente y Administrativo, Proceso de Información y Referencia, Proceso de Gestión de la Calidad y Acreditación, Proceso de Responsabilidad Social Universitaria y Proceso de Gestión de Formación Integral.

- La función sistémica de auditoria y monitoreo, sistema 3*, está compuesto por el Proceso de Auditoria y Monitoreo. 
- La función sistémica de adaptación e inteligencia, sistema 4, está compuesto por el Proceso de Adaptación e Inteligencia.

- La función sistémica de identidad y política, sistema 5, está compuesto por el Proceso de Identidad y Política.

\subsection{Mapa de procesos de la Facultad}

El mapa de procesos de la Facultad de Ingeniería en el mismo sentido de sistema viable, tiene el propósito brindar el servicio de formación profesional e investigación, a través de la enseñanza aprendizaje, investigación, extensión cultural y proyección social para coadyuvar al desarrollo regional y nacional. Para cumplir dicho propósito desarrolla a su interior las seis funciones sistémicas mediante tres macro procesos, denominados con respecto a los sistemas I, 2, 3, 3*, 4, 5: Formación Integral (SI), Cohesión y Monitoreo (S2, S3, S3*) y Dirección y Adaptación (S4 y S5) (ver Figura 2).

El Macro proceso de Formación Integral, encargado de llevar a cabo los procesos para cumplir el propósito de la Facultad, lo integran los procesos: Enseñanza - Aprendizaje, Investigación, Extensión Cultural y Proyección Social.

El Macro proceso de Cohesión y Monitoreo, encargado de llevar a cabo la negociación de recursos, gestión del talento humano, planes operativos y directivas; además, recoge información sobre el análisis misional y entrega informes de auditorías.

El Macro proceso de Dirección y Adaptación, encargado de recibir del macro proceso de cohesión y monitoreo la información del aquí y ahora de la Facultad, y por parte del entorno las tendencias y novedades, como resultado de esta interacción de información entrega estudios prospectivos. También es el encargado de proporcionar la visión, misión y lineamientos de políticas para la Facultad.

Figura 2. Mapa de procesos de la Facultad de Ingeniería

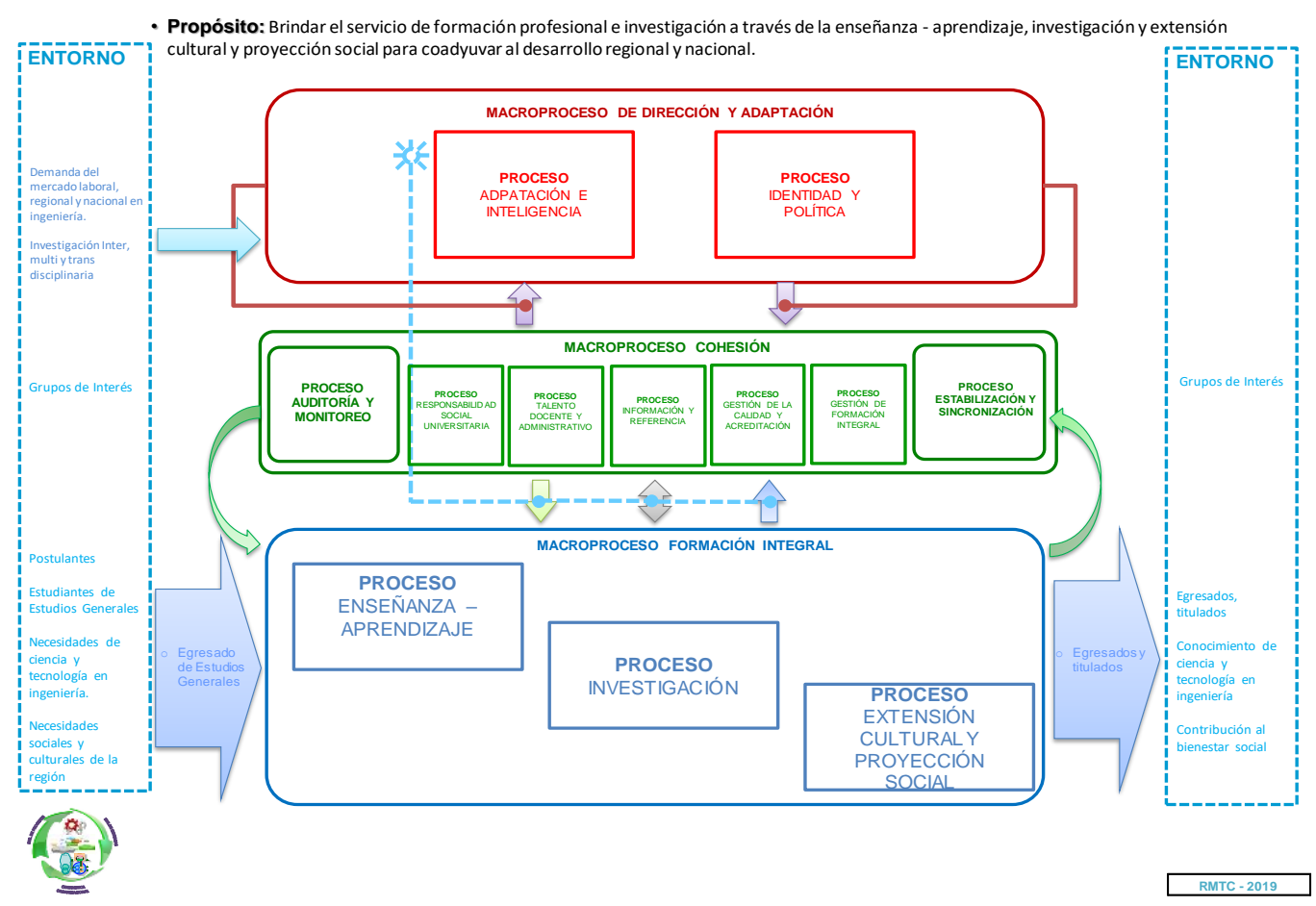




\section{Discusión}

El diseño organizacional mediante el enfoque sistémico-cibernético, por un lado, esclarece el sentido y propósito de la organización, asimismo, el "ser organización"; por otro, contribuye considerando en el diseño las seis funciones sistémicas: operación, coordinación, cohesión, auditoría, adaptación, e identidad - política, necesarias para la viabilidad de la organización. Sin embargo, la difusión y utilidad del enfoque sistémico-cibernético está restringida en algunas organizaciones y ámbitos académicos.

El sistema de gestión son elementos interrelacionados que permiten desarrollar el "fin organizacional", es decir, es un despliegue formal de los "medios organizacionales". El sistema de gestión, casi siempre ha sido relacionado al cumplimiento de requerimientos en base alguna norma ISO, ejemplo ISO 900I:20I5, dejando de lado el sentido y significado de la organización. El enfoque sistémico-cibernético, insta y considera en forma seria el "ser organización" con su característica compleja y sistémica, ello asegura, el diseño de su sistema de gestión pertinente y en contexto.

\section{Conclusiones}

El enfoque sistémico-cibernético aborda la organización esclareciendo sus fines y medios organizacionales deseables e idealizados. Ello incide en el diseño del sistema de gestión propio y particular de la institución.

Enfatizar el valor del diseño organizacional mediante el enfoque sistémico y la cibernética organizacional, en específico el sistema de gestión basada en procesos, es reiterar la aplicación de: la construcción del modelo del sistema viable, análisis de funciones sistémicas, diseño de procesos agrupados en funciones sistémicas y el mapa de procesos.

\section{Referencias bibliográficas}

Ackoff, R. (2006). Why Few Organizations Adopt Systems Thinking. Systems Research and Behavioral Science, 23(5), 705-708. https://doi.org/|0.1002/sres.79|

Beer, S. (1985). Diagnosing the System for Organizations (Isd ed.). Wiley.

Bretz, L., Könemann, U., Anacker, H., \& Dumitrescu, R. (2020). A contribution to the design of organizational structures suitable for Systems Engineering. Procedia CIRP 9I, I0I-I06. https://doi.org/10.1016/j.procir.2020.02.154

Checkland, P. (2019). Reflections on 40 years in the management field: A Parthian shot (friendly). Journal of the Operational Research Society, 70(8), I219-1223. https://doi.org/10.1080/01605682.2019.1590137

Churchman, C. W. (1968). The Systems Approach. Delacorte Press.

Churchman, C. (1995). Ethics and Science. Systems Research, I2(4), 267-27I. https://doi.org/I0.1002/sres.3850I20406 
Collopy, F. (2019). Why the Failure of Systems Thinking Should Inform the Future of Design Thinking. Design Issues, 97-100. https://doi.org/10.1 I62/desi_a_00538

Espejo, R., \& Reyes, A. (20I I). Organizational Systems: Managing Complexity with the Viable System Model. Springer

Flores, S., \& Ludueña, M. (1983). Teoría General de Sistemas y Cibernética, Cuaderno Nro 7. Grupo de Estudios de Sistemas Integrados.

Glanville, R. (2007). Try again. Fail again. Fail better:the cybernetics in design and thedesign in cybernetics. Kybernetes, 36(9/10), I173-1206. https://doi.org/I0.1 108/036849207/ 0827238

Hernández Sampieri, R., Fernández Collado, C., \& Baptista Lucio, P. (20I4). Metodología de la Investigación. Mc Graw Hill Education.

Hurtado de Barrera, J. (2010). Metodología de la Investigación. Guía para la comprensión holística de la ciencia. Quirón.

Jackson, M. (2019). Critical Systems Thinking and the Management of Complexity. John Wiley \& Sons Ltd.

Ley General de Educación, Ley Nro. 28044 [MINEDU]. (28 de Julio de 2003). Lima, Lima, Perú.

Decreto Supremo No 01 2-2020-MINEDU del 2020 [MINEDU]. Política Nacional de Educación Superior y Técnico-Productiva.(3I de agosto del 2020). Lima, Lima, Perú

Nadler, G. (1985). Systems methodology and design. IEEE Transactions on Systems, Man, and Cybernetics, 15(6), 685-697. https://doi.org/10.1 I09/TSMC.1985.6313452

Pérez Ríos, J. (2012). Design and Diagnosis for Sustainable Organizations The Viable System Method. Springer.

Saeed Rezk, S., \& Gamal, S. (2019). An Organizational Cybernetics Framework for Designing a Viable Higher Education System. Systemic Practice and Action Research, 33, 703-724. https://doi.org/ I0.1007/s I I 213-019-09505-9

Schwaninger, M. (2009). Intelligent Organizations. Germany: Springer.

Taipe Castro, R. (2009c). Diseño de la universidad basado en el enfoque de sistema viable. Tomo III. UNCP.

Taipe Castro, R. (2018). Estudio sistémico interpretativo de una institución de educación superior universitaria para el diseño de un sistema de gestión. UNCP.

Taipe Castro, R. M. (2009a). Identificación y análisis de la UNCP Tomo I. UNCP.

Taipe Castro, R. M. (2009b). Diagnóstico de la universidad basado en el enfoque de sistema viable Tomo II. UNCP.

UNESCO. (2017). Six ways to ensure higher education leaves no one behind. Global Education Monitoring. 
Vriens, D., \& Achterbergh, J. (20II). Cybernetically sound organizational structures I: de Sitter's design theory. Kybernetes, 40(3/4), 405-424.

https://doi.org//0.1 I08/0368492 I I I I 33656

\section{Financiamiento}

Ninguno.

\section{Conflicto de intereses}

El artículo no presenta conflicto de intereses.

\section{Contribución de autores}

Taipe-Castro, Robensoy: Investigador y redactor del presente artículo. 